\title{
Finger creases lend a hand in Kabuki syndrome
}

Caroline Michot ${ }^{\mathrm{a}^{*}}$, Carole Corsini $\mathrm{b}^{\mathrm{b}^{*}}$, Damien Sanlaville ${ }^{\mathrm{c}}$, Clarisse Baumann $^{\mathrm{d}}$, Annick Toutain ${ }^{\mathrm{e}}$, Nicole Philip $^{f}$, Tiffany Busa $^{f}$, Muriel Holder ${ }^{g}$, Laurence Faivre ${ }^{h}$, Sylvie Odent', Marie-Ange Delrue, Marianne Till $^{c}$, Marie-Line Jacquemont ${ }^{k}$, Marie-Pierre Cordier $^{c}$, Alice Goldenberg', Elodie Sanchez ${ }^{b}$, Eudeline Alix $^{c}$, Sylvain Poisson ${ }^{a}$, Honorine Kayirangwa ${ }^{a}$, Didier Lacombe', Brigitte Gilbert-Dussardier ${ }^{m}$, Anna Pelet $^{\mathrm{a}}$, Joëlle Roume ${ }^{\mathrm{n}}$, Aurélia Jacquette ${ }^{o},{\text { Bertrand } \text { Isidor }^{\mathrm{p}} \text {, Fabienne Giuliano }}^{q}$, Lydie Burglen ${ }^{r}$, Mélanie Fradin ${ }^{i-s}$, Elise Schaefer ${ }^{s}, Y_{\text {ves Alembick }}^{\text {s }}$, Bérénice Doray ${ }^{\mathrm{s}}$, Anne Moncla $^{\mathrm{f}}$, Delphine Héron ${ }^{\circ}$, Marjolaine Willems ${ }^{b}$, Lucile Pinson ${ }^{b}$, Kim Hanh Le Quan Sang ${ }^{a}$, Martine Le Merrer ${ }^{a}$, Valérie CormierDaire $^{\mathrm{a}}$, Pierre Sarda ${ }^{\mathrm{b}}$, Jeanne Amiel ${ }^{\mathrm{a}}$, Stanislas Lyonnet ${ }^{\mathrm{a}}$, David Geneviève ${ }^{\mathrm{b}}$.

a1 Département de Génétique, Unité Inserm U781, Institut Imagine, Hôpital Necker enfants Malades, CLAD Ile de France, Paris,

b Département de Génétique Médicale, CHRU Montpellier, Université Montpellier 1, CLAD Sud Languedoc-Roussillon,

c Département de Cytogénétique, Hospices civil de Lyon, Centre des neurosciences, Tiger, Université Claude Bernard Lyon 1, CLAD centre-est, Lyon,

d Département de Génétique, Hôpital Robert Debré, CLAD Ile de France, Paris

e Service de Génétique, Hôpital Bretonneau, CLAD Ouest, Tours

f Département de Génétique Médicale, Hôpital de la Timone, CLAD Sud-PACA, Marseille, g Service de Génétique Clinique, Hôpital Jeanne de Flandre, CLAD Nord, Lille

h Centre de Génétique, Hôpital d'enfant, CLAD Est, Dijon,

i Service de Génétique médicale, Hôpital Sud, CLAD Ouest, Rennes,

j Service de Génétique, Hôpital Pellegrin, CLAD Sud-Ouest, Bordeaux,

k Service de Génétique, Hôpital saint Pierre, GH Sud Réunion, lle de la Réunion, I Service de Génétique, CHU de Rouen, Rouen, 
m Service de Génétique, CHU La Milétrie, CLAD Ouest, Poitiers,

n Service de Génétique, Hôpital Poissy-saint Germain, Poissy,

o Département de Génétique, CHU La Pitié-Salpêtrière, CLAD Ile de France, Paris

p Service de Génétique, CHU Nantes, CLAD Ouest, Nantes,

q Service de Génétique, CHU Lenval, CLAD Sud-PACA, Nice,

r Service de Génétique, CHU Trousseau, CLAD Ile de France, Paris,

s Service de Génétique, Hôpital de Hautepierre, CHU Strasbourg, CLAD Est, Strasbourg, France

* These authors are equally contributors

Corresponding author:

Professor David Genevieve, Md, PhD

Département de Génétique Médicale, CHRU Arnaud de Villeneuve

371 avenue du doyen Gaston Giraud, 34000 Montpellier, France

Tel: +33 (0)4 67336564

Fax: +33(0)467336052

Email: d-genevieve@chu-montpellier.fr

Keywords: Kabuki syndrome, MLL2, novel clinical feature, finger crease, attenuation

Running title: Finger creases in Kabuki syndrome 


\section{Abstract}

Kabuki syndrome (KS) is a rare syndrome associating malformations with intellectual deficiency and numerous visceral, orthopedic, endocrinological, immune and autoimmune complications.

The early establishment of a diagnostic of KS leads to better care of the patients and therefore prevents complications such as perception deafness, severe complications of auto-immune diseases or obesity.

However, the diagnosis of KS remains difficult because based on the appreciation of facial features combined with other highly variable features.

R3 We describe a novel sign, namely the attenuation and/or congenital absence of the IPD crease of the third and fourth fingers associated with limitation of flexion of the corresponding joints, which seems to be specific of KS and could help the clinician to diagnose KS. 


\section{Introduction}

Kabuki syndrome (KS, MIM 147920, also named Niikawa-Kuroki syndrome) is a multiple congenital anomaly-Intellectual disability (ID) syndrome characterized by a distinctive facial phenotype, including high arched eyebrows with flaring or interruption in the middle, long palpebral fissures with eversion of the third lateral part of the lower eyelid, short nose with short columella and depressed nasal tip, long philtrum, small mouth with pillowed lower lip and large prominent or malformed ears. Numerous other manifestations such as visceral malformations, non-specific skeletal features such as scoliosis, fetal fingertip pads, postnatal short stature with overweight, endocrinological dysfunction and immunological problems are also observed. Recently, heterozygous mutations in the MLL2 [1] gene and deletion or point mutation in the KDM6A [2-3] gene have been indentified in about $70 \%$ of the patients fulfilling clinical diagnostic criteria. Despite a wide range of clinical features and the recent identification of the molecular basis of KS, the diagnosis of KS remains based on the clinician's identification of the facial features making this diagnosis difficult in particular when the features are subtle.

We have identified a novel clinical sign, namely the attenuation and/or congenital absence of the distal interphalangeal (DIP) flexion crease on the third and fourth joint of fingers with limitation of flexion of the linked joints in patients with KS, which could help the clinician in the diagnostic of patients suspected to have KS. 


\section{REPORT}

We have collected clinical and biological data for patients diagnosed with KS in accordance with the French research program PHRC AOM-09-070 and performed molecular screening for either mutation or deletion in the MLL2 and KDM6A genes. The project was approved by the human ethic commity entitled 'Comité de Protection des Personnes Ile de France II'. The diagnosis of KS has been performed clinically, based on facial and other clinical features, by at least more than 5 clinical geneticists (figure 1). We analysed information concerning orthopaedic features such as scoliosis, abnormality or dislocation of the hip joint, patellar dislocation, hands and skeletal x-rays from the collection of data.

In KS during early infancy hands are short and fingers are stubby. Brachymesophalangy of the fifth finger and persistence of fetal fingertip pads are frequently observed. Later, the fingers are extended and gradually became finer.

During follow up of the patients from our series, we identified a novel clinical feature, which seems to be specific for Kabuki syndrome, namely the attenuation and/or congenital absence of the distal interphalangeal crease of the third and fourth fingers in several patients (Figure 2a, and magnified view of figure $2 a)$.

The attenuation and/or congenitally missing of the DIP crease on the volar side can concern only the third or the fourth finger, or both, and is usually bilateral but can be asymmetrical. Interestingly, some patients showed attenuation of the IPD creases but no MLL2 or KDM6A mutation. However, search for exonic deletions has not been performed in these patients. We focus on this novel sign in the 82 KS patients from our series with available data regarding the palmar and finger creases and with an identified mutation in the MLL2 gene.

The status of attenuation and/or congenital absence of the DIP crease has been established on available pictures or on the clinical description from the clinicians from 82 patients with KS and MLL2 mutations. 
The attenuation and/or congenital absence of the DIP crease is more frequently observed on the

R7

R8

fourth finger ( 8 patients on 82 for the third digit versus 41 patients on 82 for the fourth) and is found most frequent on the right hand. This clinical feature is present in about $50 \%$ of the patients from our series with mutation in the MLL2 gene (41/82). However, this feature seems more frequently observed in older patients ( $36 / 60=60 \%$ of KS patients after the age of 10 years, table1). Moreover, there is no statistical association between the nature of the mutations (truncating versus non truncating) and the absence of the DIP crease $\left(p=0,65-\chi^{2}\right)$.

In addition to the attenuation and/or congenital absence of the DIP crease on the volar side of the third and fourth fingers, we also observed limitation of movement in the interphalangeal joint underlying the disappeared and/or congenital absence crease. This limitation of the interphalangeal joint is associated with a peculiar shape of the second and third phalanges, which seem to tighten from the proximal interphalangeal joint and then become thinner (Figure 2b). However, hand x-rays are normal and show neither a decrease in the size of the interphalangeal joint corresponding to the joint stiffness nor morphological changes of the phalanges (Figure 3).

The DIP crease seems to wear off progressively during life. This clinical feature, namely the attenuation and/or congenital absence of the DIP crease of the volar side of the third and fourth fingers, is usually observed between the ages of 7 to 8 years. A statistically significant association between the absence of the DIP crease and the age below/above 10 years old $(p=0,0022)$ was observed and seems to be consistent as a cut-off for the apparition of this anatomical landmark for KS. However, it can be observed earlier and seems to be correlated with the severity of the neurological manifestations.

\section{DISCUSSION}

Examination of the patient's hands provides important information for the clinician attempting to diagnose genetic syndromes such as Kabuki syndrome because of the observation of abnormal 
dermatoglyphs and persistence of fetal fingertip pads. However, despite the discovery of the molecular basis of this syndrome the diagnosis of KS remains difficult because it is based on nonspecific clinical features. We report herein what we believe to be novel clinical signs specific to KS diagnosis, namely the attenuation and/or congenital absence during the course of the disease of IPD finger creases on the third and fourth digits of the hands, associated with flexion limitation of the corresponding joints.

Palmar and digital flexion creases develop between 8 and 13 weeks of fetal development [4]. Distal interphalangeal flexion creases appear concurrently with the appearance of the digital pads by about 9 weeks of gestation in humans [4]. Data regarding mechanisms responsible for the appearance of palmar and digital creases are controversial and 2 opposing hypotheses exist, namely either palmar and digital creases are secondary features caused by flexion movements or creases develop independently of palmar and finger movements.

Palmar creases overlying the various osseous structures of the hands are important anatomical landmarks which, when abnormal, constitute a noteworthy clinical feature underlying numerous genetic disorders.

Indeed, abnormal dermatoglyphs as well as abnormal palmar and finger creases are observed in numerous genetic disorders such as chromosomal disorders including Down and Turner syndromes. The absence of fingerprints could be inherited in an autosomal-dominant fashion through mutations in the skin-specific isoform of SMARCAD1 [5]. Other syndromes such as Naegeli-FranceschettiJadassohn syndrome (MIM 161000), and dyskeratosis congenita (MIM 305000) also present abnormal development of epidermal ridges. However, interphalangeal finger and palmar creases seem to be normal in patients with SMARCAD1 mutations.

In addition, abnormal palmar and plantar creases are observed in spondylocheiro dysplastic EhlersDanlos syndrome due to mutations in the SLC39A13 gene [6].

Absence of creases of the dorsal side of fingers overlying the distal interphalangeal joints is observed in Nail-patela syndrome with or without nail dysplasia. This feature is usually associated with 
hypoplasia of the distal phalanges and does not seem to be linked to abnormal movement during the fetal period [7].

Congenital absence of finger creases can be observed in genetic disorders expressed early in foetal life or in cases where environmental hazards act prior to the development of fetal creases. Indeed, hand malformations or alterations of the form or function of the hand, such as neuromuscular disorders, may affect the developing flexion creases and support the hypothesis that creases are the consequence of flexion movements. Indeed, missing palmar and finger creases on the volar side are frequently observed in patients with neuromuscular disorders with prenatal manifestations and are linked to immobility during the fetal life period.

Congenital absence of creases on the volar side of fingers has also been observed over affected joints as described in distal symphalangism or in patients with $4 q 31$ deletion [8-9]. Usually the absence of creases is associated with the absence of interphalangeal joints in these syndromes.

Missing creases have also been observed in severe brachymesophalangy as seen in trisomy 21 for example. Indeed, in a series of 108 patients with trisomy 21, 14 patients presented with a single interphalangeal crease of the volar side of the fifth finger but normal inter-phalangeal joints on hand x-rays [10]. However, in this case the creases had been missing since birth or before birth - they did not disappear during the course of the disease. Interestingly, in the same report one patient from a series of 50 additional patients with severe ID, presented with absence of the distal interphalangeal flexion creases of the third and fourth fingers. Hand X-rays and joint function were described as normal. Because this description precedes the delineation of KS, it is tempting to speculate that this patient actually had KS. Unfortunately, no frontal or lateral view of the patient was available in the report.

Missing finger creases seems not to be observed in normal subjects [10]. However, an extensive review of the literature reveals the report of two families without DIP creases of the second to fifth fingers and limited flexion of these fingers, inherited as a dominant trait [11-12]. Except for the index case reported by Fried and Mundel [11], no other members of this family presented with ID. In 
addition, the pictures of the hands available in these reports revealed a similar shape of the DIP joint with tightening of the joint rather than an enlargement.

R1 We believe that the fine motor difficulties observed in Kabuki syndrome cause limitation of the use of the third and fourth fingers, thus leading to joint stiffness, and that this is the most-likely mechanism responsible for the attenuation of the DIP crease of the third and fourth fingers. We suggest that this feature could be a novel anatomical landmark for KS and could help the clinician make this diagnosis. However, this clinical sign appears late in infancy and cannot help the clinician in the early management of patients with KS.

\section{AKNOWLEDGMENTS}

We thanks the families as well as the French Kabuki association (http://asso.orpha.net/ASK/cgi-bin/) for their participation to this study

\section{FUNDINGS}

Part of this work has been supported by French ministry of health (Programme Hospitalier de Recherche Clinique national AOM 07-090), Fondation Maladies Rares, and the French Kabuki association. 


\section{LEGEND OF FIGURES}

Figure 1: Front and lateral view of 4 patients with KS and MLL2 mutation.

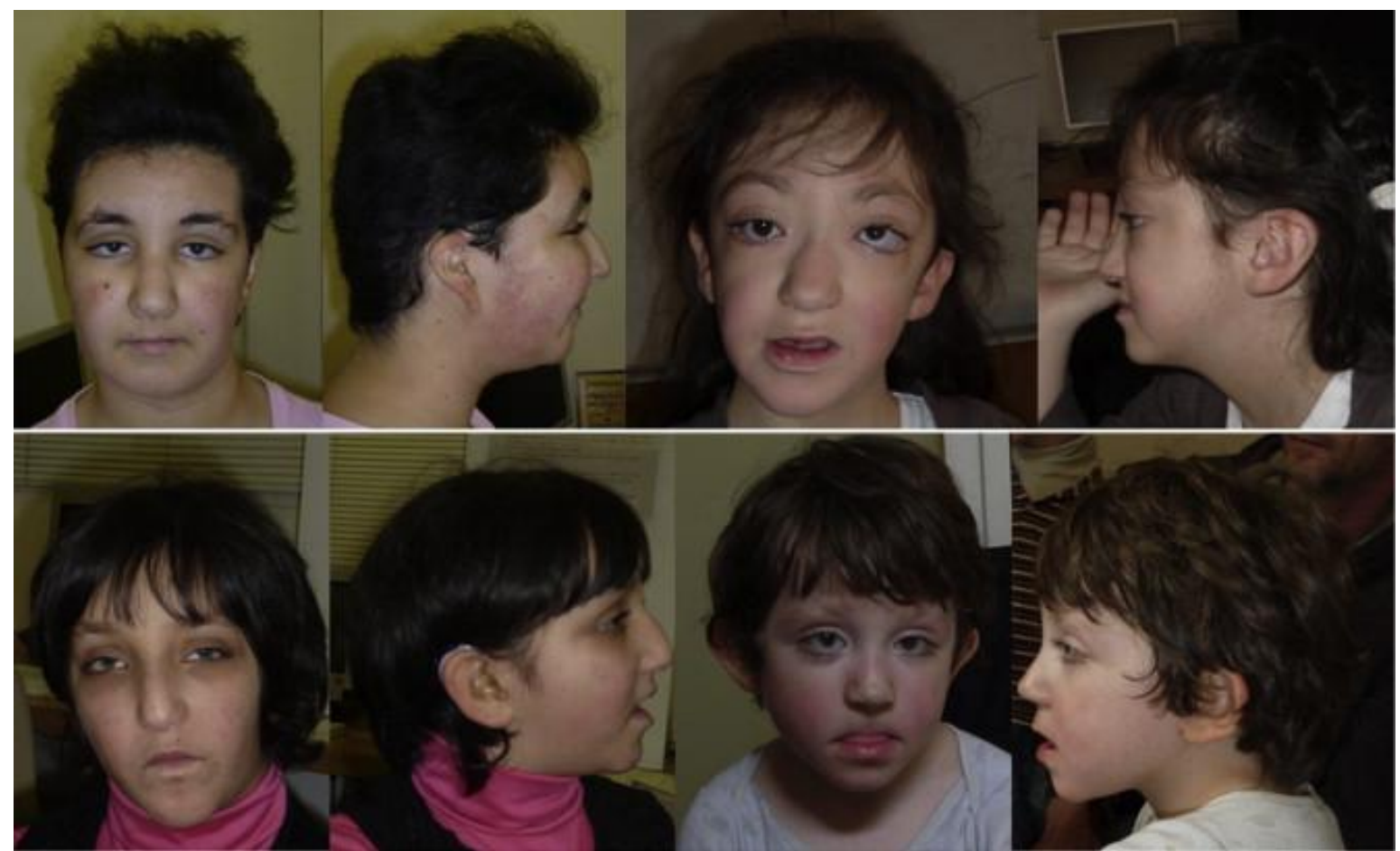


Figure 2: Attenuation of the IPD creases of third and fourth fingers in KS patients in a) patient NCK020 at 16 months and 10 years of age, b) Attenuation and/or congenital absence of the IPD creases of third and fourth fingers in patient LAR003 at 3 years and 11 years of age, and c) in patient TOU002 at 6 years $1 / 2$ and 24 years of age.

a
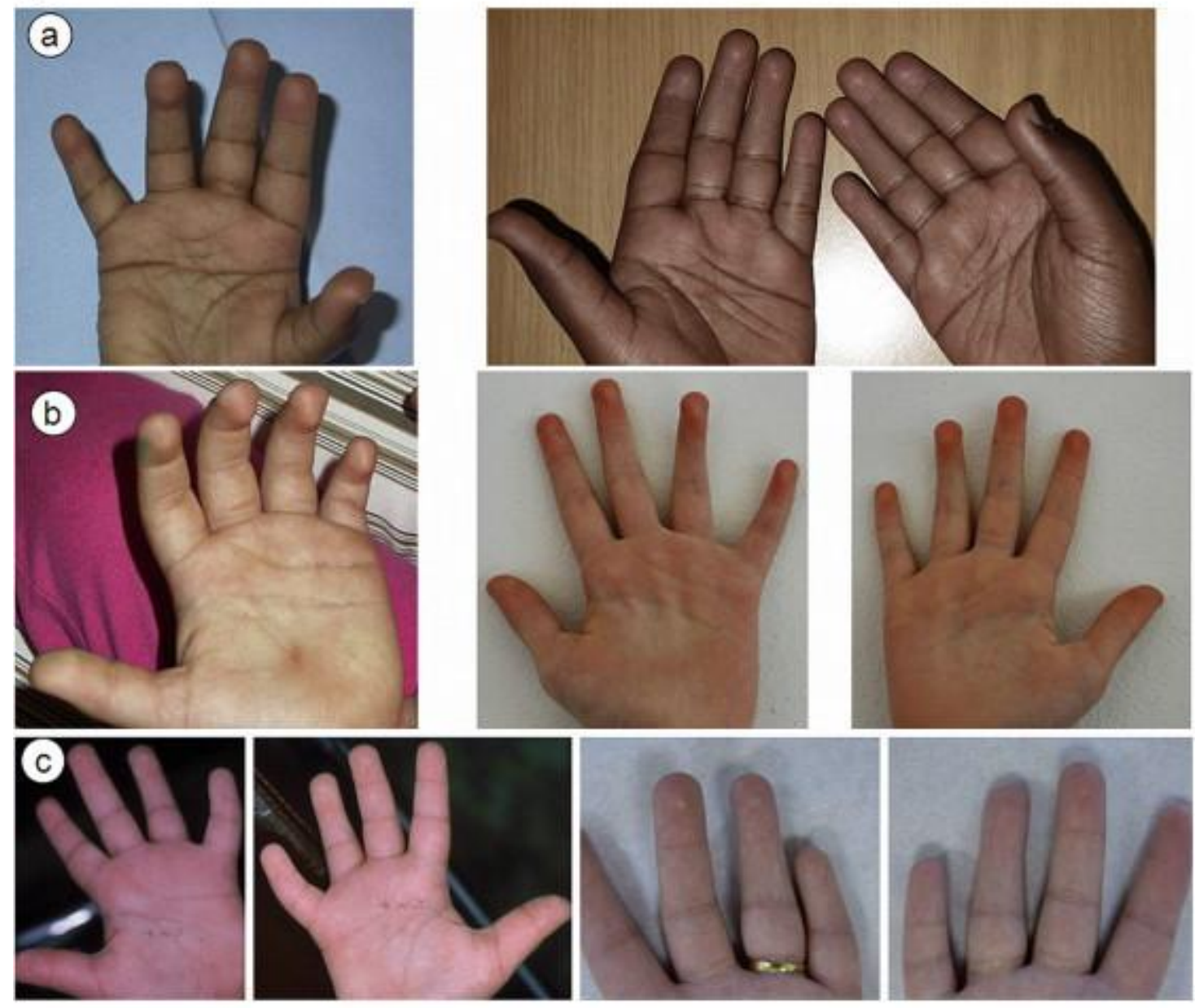

b

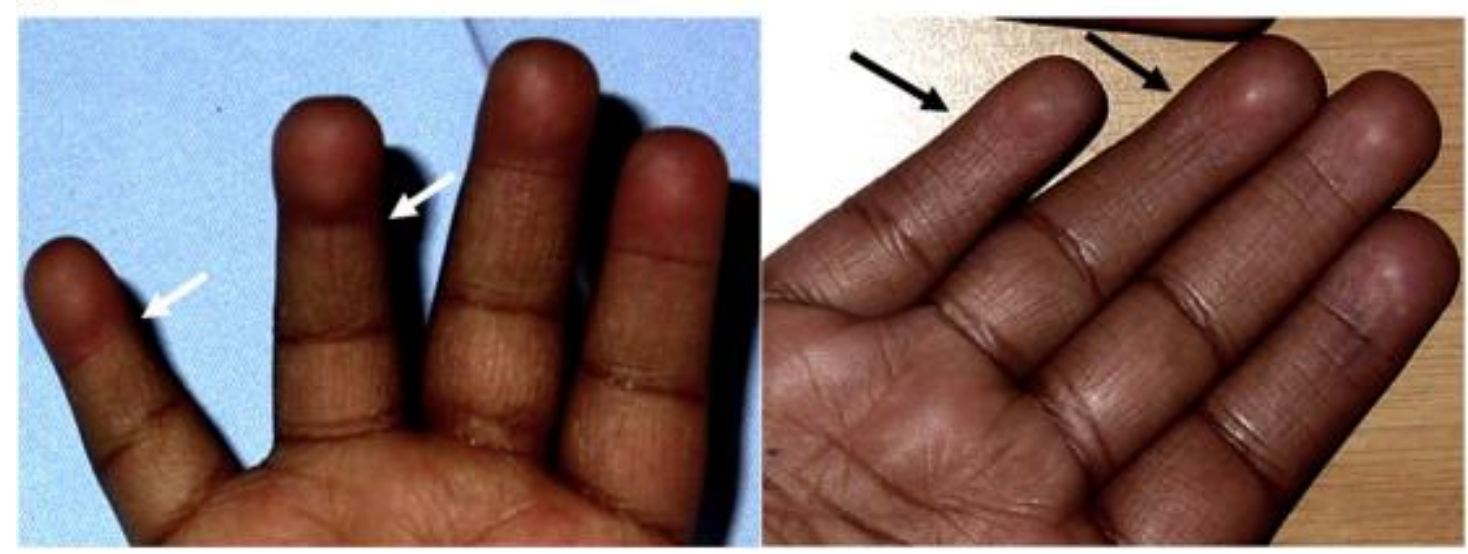


Figure 3 Note the peculiar shape of the IPD with tightening of the joint rather than an enlargement in patients NCK025, RDB008, NCK015 and NAT001 at 15, 11 1/2, 14 1/2 and 29 years of age respectively.

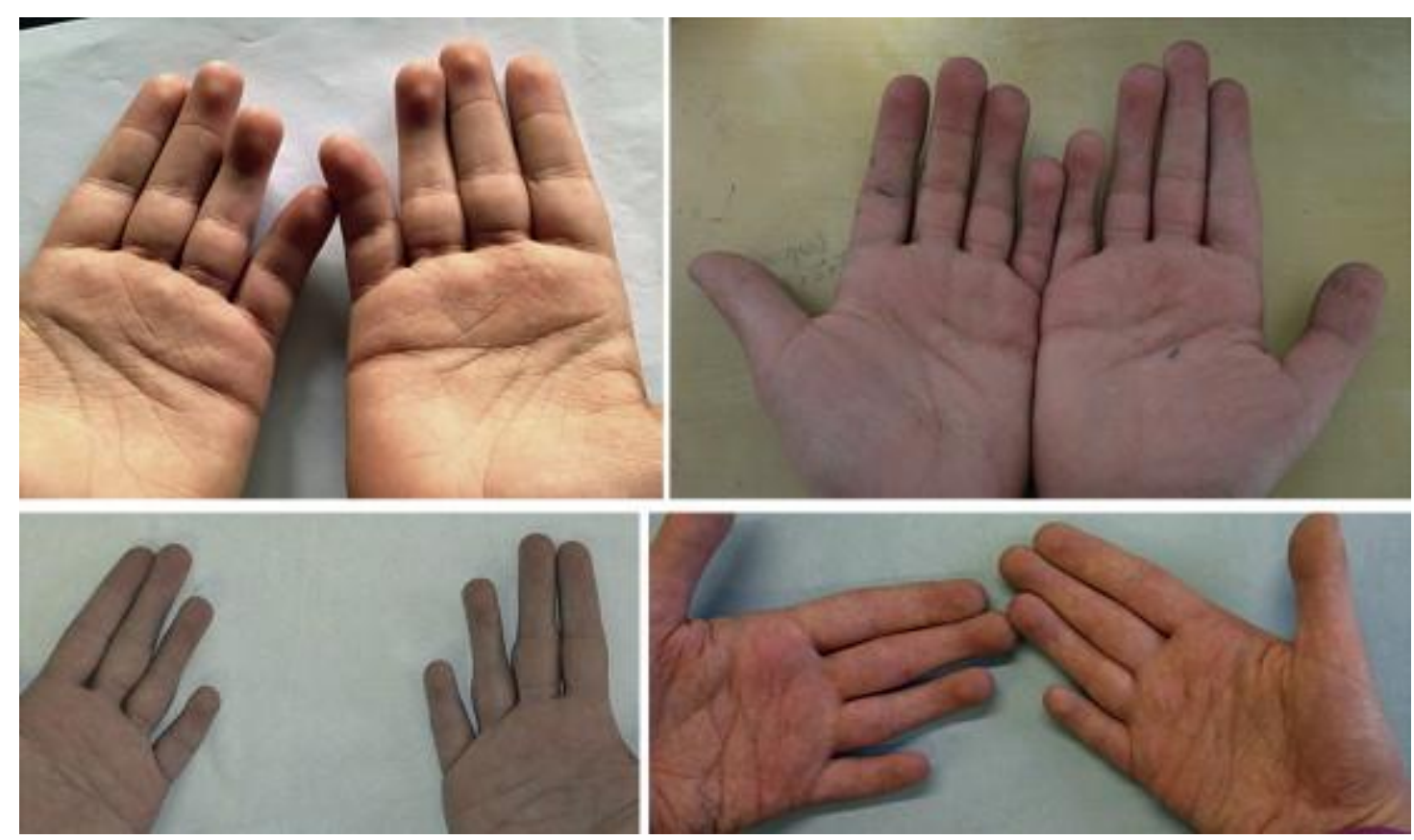

Figure 4: Normal skeletal x-rays of the left hand of the patients MON001, NCK007 and NCK018 with vanished volar crease of the IPD of the third and/or the fourth fingers at 11,14 and 12 years of age respectively.

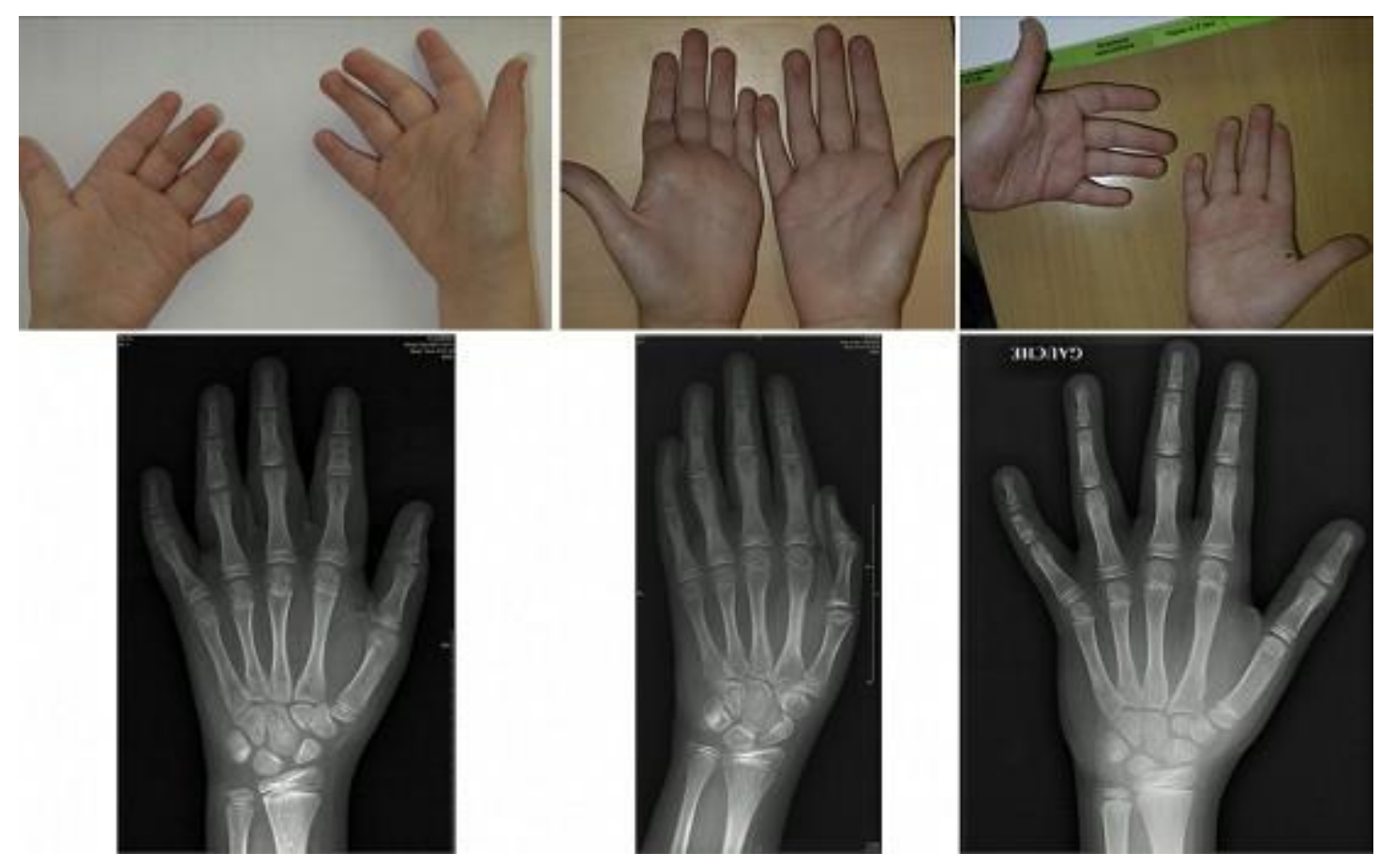




\section{REFERENCES}

[1] Ng SB, Bigham AW, Buckingham KJ, Hannibal MC, McMillin MJ, Gildersleeve HI, Beck AE, Tabor HK, Cooper GM, Mefford HC, Lee C, Turner EH, Smith JD, Rieder MJ, Yoshiura K, Matsumoto N, Ohta T, Niikawa N, Nickerson DA, Bamshad MJ, Shendure J. Exome sequencing identifies MLL2 mutations as a cause of Kabuki syndrome. Nat Genet. 2010 Sep;42(9): 790-3.

[2] Lederer D, Grisart B, Digilio MC, Benoit V, Crespin M, Ghariani SC, Maystadt I, Dallapiccola B, Verellen-Dumoulin C. Deletion of KDM6A, a Histone Demethylase Interacting with MLL2, in Three Patients with Kabuki Syndrome. Am J Hum Genet. 2012; 90(1): 119-24.

[3] Miyake N, Mizuno S, Okamoto N, Ohashi H, Shiina M, Ogata K, Tsurusaki Y, Nakashima M, Saitsu H, Niikawa N, Matsumoto N. KDM6A point mutations cause Kabuki syndrome. Hum Mutat. 2013; 34(1): 108-10.

[4] Kimura S. Embryologic development of flexion creases. Birth Defects Orig Artic Ser. 1991; 27(2): 113-29.

[5] Nousbeck J, Burger B, Fuchs-Telem D, Pavlovsky M, Fenig S, Sarig O, Itin P, Sprecher E. A mutation in a skin-specific isoform of SMARCAD1 causes autosomal-dominant adermatoglyphia. Am J Hum Genet. 2011; 89(2): 302-7.

[6] Giunta C, Elçioglu NH, Albrecht B, Eich G, Chambaz C, Janecke AR, Yeowell H, Weis M, Eyre DR, Kraenzlin M, Steinmann B. Spondylocheiro dysplastic form of the Ehlers-Danlos syndrome--an autosomal-recessive entity caused by mutations in the zinc transporter gene SLC39A13. Am J Hum Genet. 2008; 82(6): 1290-305. 
[7] Itin PH, Eich G, Fistarol SK.Missing creases of distal finger joints as a diagnostic clue of nail-patella syndrome. Dermatology. 2006; 213(2): 153-5.

[8] Poush JR. Distal symphalangism: a report of two families. J Hered. 1991; 82(3): 233-8.

[9] Davis JM, Clarren SK, Salk DJ. Brief clinical report: the del(4) (q31) syndrome- a recognizable disorder with atypical Robin malformation sequence. Am J Med Genet. 1981; 9(2): 113-7.

[10] Aue-Hauser G. Flexion creases on fingers. Birth Defects Orig Artic Ser. 1979; 15(6): 697-720.

[11] Fried $\mathrm{K}$ and Mundel $\mathrm{G}$. Absence of distal interphalangeal creases of fingers with flexion limitation, J Med Genet. 1976; 13: 127-130.

[12] Lambert D, Nivelon-Chevallier A, Chapuis JL. Absence of distal interphalangeal fold causing difficulty in extending fingers. J Med Genet. 1977; 14(6): 466-7. 